PALEO

Revue d'archéologie préhistorique

$30-2 \mid 2020$

Varia

\title{
Nicole Pigeot
}

(1950-2019)

\section{François Bon}

\section{(2) OpenEdition}

\section{Journals}

Édition électronique

URL : http://journals.openedition.org/paleo/5018

DOI : $10.4000 /$ paleo.5018

ISSN : 2101-0420

Éditeur

SAMRA

\section{Édition imprimée}

Date de publication : 1 septembre 2020

Pagination : 15-16

ISSN : 1145-3370

\section{Référence électronique}

François Bon, « Nicole Pigeot », PALEO [En ligne], 30-2 | 2020, mis en ligne le 29 septembre 2020, consulté le 26 novembre 2020. URL : http://journals.openedition.org/paleo/5018 ; DOI : https:// doi.org/10.4000/paleo.5018

\section{(ब) $\odot \Theta$}

PALEO est mis à disposition selon les termes de la licence Creative Commons Attribution - Pas d'Utilisation Commerciale - Pas de Modification 4.0 International. 


\section{NICOLE PIGEOT $(1950-2019)$}

Son regard derrière des lunettes légèrement fumées, le grain de sa voix et son rire, sont gravés dans la mémoire de ceux qui l'ont connue, comme le rythme bien à elle de ses gestes, tandis qu'elle aspire par exemple une nouvelle bouffée de cigarette - plaisir dont elle sut d'ailleurs se priver, à la grande admiration de toutes celles et ceux qui l'avaient vu en consumer beaucoup.

Nicole Pigeot a été et reste l'une des personnalités scientifiques et intellectuelles emblématiques de ce que l'on a pu désigner comme « une nouvelle vague » dans le domaine des études préhistoriques. Initiée il y a une quarantaine d'années et prônant une approche nouvelle de la culture matérielle des sociétés passées, celle-ci a permis de porter un tout autre regard sur l'humanité préhistorique. Au service de cette approche technologique - dont les prémices remontent à Leroi-Gourhan voire au-delà (et dont Jacques Tixier récemment disparu fut ensuite un promoteur déterminant) -, un bouquet de thèses fondatrices émergea au cours des années 1980. Parmi elles, celle de Nicole Pigeot demeure aujourd'hui, trente ans plus tard, un texte majeur, l'une des principales références dans ce courant de recherches dont elle fut une pionnière (Pigeot 1987). S'il faut d'un mot résumer l'apport d'un travail prenant comme terrain d'application la taille de la pierre dans l'une des unités d'habitation magdaléniennes d'Etiolles (Essonne), c'est celui de la sociologie des groupes préhistoriques. S'appuyant sur leurs savoir-faire afin de révéler le rôle de l'apprentissage et l'importance de l'acquisition des techniques dans les sociétés préhistoriques, Nicole Pigeot a conçu un cadre méthodologique profondément revisité autour d'un thème résonnant d'ailleurs avec son propre engagement professionnel : celui, justement, de la transmission. Un véritable discours sur la méthode, dans tous les sens du terme.

Nicole Pigeot a été en effet une grande enseignante, s'attachant depuis ses quartiers parisiens à former toute une génération de futurs préhistoriens - des archéologues bientôt disséminés aux quatre coins de la France, et bien au-delà. Remarquons au passage que, dans ces années 1980-1990 - c'était il n'y a pas si longtemps -, tandis que la plupart des laboratoires et chaires de Préhistoire étaient en Province tenus par des hommes, ce sont pour beaucoup des femmes qui, en région parisienne, jouèrent alors un rôle essentiel - et voici aussi, sans doute, l'un des héritages auquel elle contribua au premier chef, celui d'un précieux renouvellement de notre milieu et des perspectives scientifiques qu'il engendre, ce qui touche bel et bien au sens profond offert par ces mêmes sciences.

C'est ainsi qu'elle dirigea une trentaine de thèses, lesquelles allaient contribuer après ses propres apports à l'essor de la technologie lithique et promouvoir une approche similaire appliquée aux matières osseuses. Plus tard, lorsque le thème des relations Homme / Animal devint

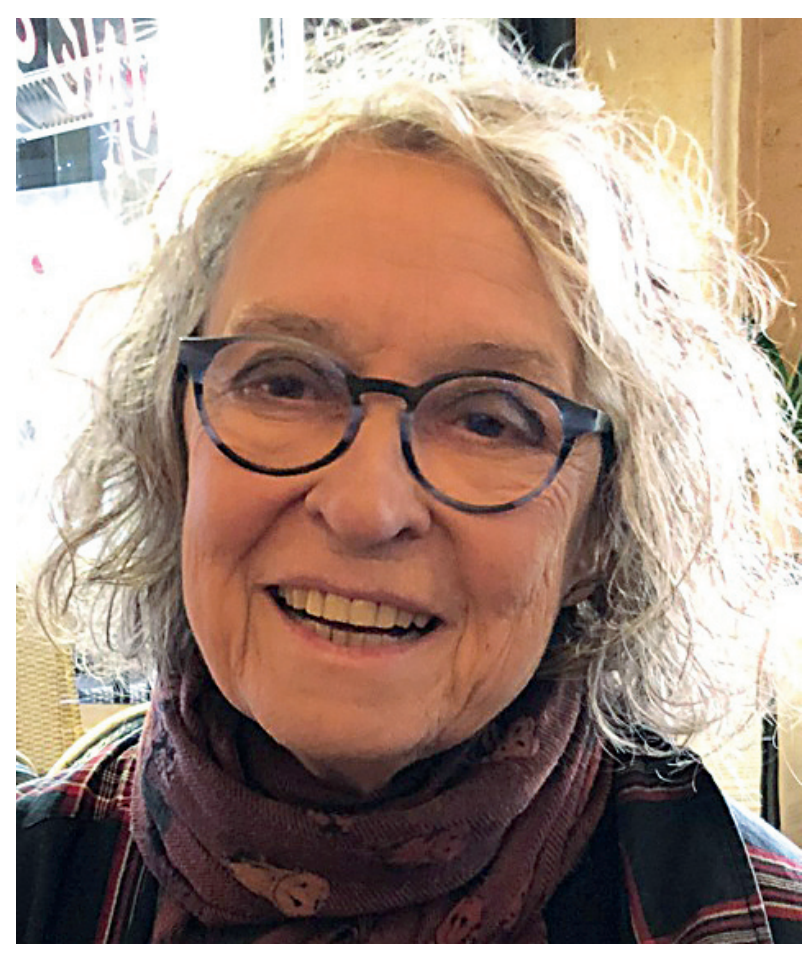

Nicole Pigeot (coll. Privée)

central dans les études préhistoriques (depuis l'industrie osseuse donc, mais aussi bien sûr depuis l'archéozoologie proprement dite et ceci sans oublier la place de l'Animal dans les productions symboliques paléolithiques), il bénéficia de son regard et de son accompagnement auprès de collègues et de doctorants travaillant sur ces sujets. Quant aux terrains d'application, ils furent très larges dans le temps comme dans l'espace. Si le Paléolithique supérieur d'Europe occidentale occupe une place de choix parmi les thèmes qu'elle encadra, bien d'autres terrains sont concernés, notamment américains, reflétant aussi son propre itinéraire de chercheuse, indissociable de son engagement d'enseignante. On soulignera encore le très fort taux de professionnalisation de ses doctorantes et doctorants, dont au moins un tiers intégrèrent le CNRS ou l'université, tandis que plusieurs autres rejoignaient l'INRAP, le ministère de la Culture ou les collectivités territoriales. Cela exprime ce qu'elle sût insuffler, le rôle structurant qui fut le sien et qui le demeure à travers elles et eux. Cela témoigne aussi de l'accompagnement chaleureux et personnalisé qu'elle offrit à beaucoup de ses étudiants jusqu'au recrutement - et au-delà.

Nicole Pigeot était une préhistorienne non conformiste. On la vit rarement se déplacer dans les congrès ou rencontres internationales; elle ne courrait pas non plus les visites organisées et excursions collectives de chantiers... Nul ne peut dire si elle s'extasiait devant des paysages calcaires, de ceux dont les karsts font habituellement le bonheur des préhistoriens. Petit clin d'oeil, on pourrait facilement l'imaginer tel un Mycroft Holmes, frère aîné du célèbre Sherlock auquel ce dernier fait appel lorsqu'il est perdu - car si Mycroft ne quitte presque jamais les fauteuils et les lumières tamisées de son club, il est doué d'un tel discernement qu'il n'a guère besoin de se 
confronter physiquement au réel pour le comprendre mieux que beaucoup d'autres, dès lors qu'on lui en rapporte des éléments intelligibles - ou plutôt qu'il rend intelligible. Mais ne nous y trompons pas : elle avait par ailleurs un sens profond et un véritable goût du terrain, plaisir archéologique dans ce qu'il a de plus sensible; un sol d'habitat préhistorique, à l'exemple de celui d'Etiolles, était pour elle une source constante d'études et de réflexions.

Nicole Pigeot a beaucoup abordé le thème de l'efficacité dans le champ des techniques, de l'intention aux gestes qui la formalisent et voici peut-être autant de clés pour une meilleure compréhension de sa personnalité. Ce qui ressort de sa trajectoire intellectuelle, de son parcours professionnel, est en effet la recherche de cohérence et l'efficacité - l'économie de moyens, dans le meilleur sens du terme. Car c'est à partir de quelques fenêtres d'observation, mais choisies avec beaucoup de discernement, et qu'elle scruta très en profondeur, qu'elle conçut une œuvre d'une telle portée. L'expérience approfondie d'un site paléolithique en particulier Etiolles, depuis 1972 - et quelques incursions comparatives en Polynésie et en Patagonie, auxquelles s'ajoutent bien sûr toutes les études de cas de provenance et de chronologie très variées, que lui soumirent, comme autant de problèmes à résoudre, ses étudiants : voici quelle fut la matière à partir de laquelle elle sut fabriquer sa propre façon d'appréhender la Préhistoire. Et toute la Préhistoire. Dans un même ordre d'idées, cette œuvre se moque bien de toute quantification bibliométrique, car sans les textes qui la composent on ne peut comprendre l'évolution des études préhistoriques au cours des 40 dernières années; et ceux-ci demeurent, et parions que ce soit pour longtemps, une puissante source d'inspiration (voir bibliographie sélective ci-dessous).

Outre le travail qu'elle consacra à l'analyse de l'apprentissage de la taille de la pierre, auquel il a déjà été fait allusion, ainsi que ses réflexions menées en collaboration avec Boris Valentin sur la matière du temps auquel se confronte tout préhistorien, un texte majeur demeure son article de 1991 publié dans les colonnes de cette revue. Là, Nicole Pigeot renouait avec le projet - si rare - d'une mise en perspective totale de l'évolution de l'Homme dans ses dimensions tout à la fois biologiques, comportementales et culturelles. Rare, car il s'agissait d'en comprendre les mécanismes, et non pas seulement d'en décrire les symptômes. Un texte qui conserve intact sa portée épistémologique et où l'on trouve ces lignes qui la définissent si bien, des mots qui continueront à accompagner toutes celles et tous ceux qui ont eu la chance de la connaître parce qu'ils auront vécu avec elle quelques-unes de leurs plus grandes émotions intellectuelles, parfois à l'improviste, au coin d'une table amicale, tandis qu'elle allumait lentement une autre cigarette : " «Débattre»... voilà sans doute le maître-mot ».

François Bon

\section{BIBLIOGRAPHIE SÉLECTIVE 1}

PIGEOT N. 1987 - Magdaléniens d'Étiolles. Économie de débitage et organisation sociale, XXIe Supplément à Gallia Préhistoire, Éditions du CNRS, Paris, 168 p. (publication de sa thèse de 1983, intitulée « Les Magdaléniens de l'unité U5 d'Étiolles. Étude technique, économique, sociale par la dynamique du débitage »).

PIGEOT N. 1988 - Apprendre à débiter des lames : un cas d'éducation technique chez des Magdaléniens d'Étiolles, in J. Tixier (éd.), Technologie préhistorique, Notes et monographies techniques, 25, CNRS, p. 63-70.

PIGEOT N. 1990 - Technical and social actors in Prehistory Flintknapping specialists and apprentices at Magdalenian Étiolles, Archaeological Review from Cambridge, 9-1, numéro spécial, Technology in the Humanities, Cambridge, p. 126-141.

PIGEOT N. 1991 - Réflexions sur l'histoire technique de l'homme: de l'évolution cognitive à l'évolution culturelle, Paleo, 3, p. 167-200. (Nourrit de son mémoire d'HDR présenté en 1992 et intitulé Entre Nature et Culture : valeur heuristique de la technologie lithique par des approches systémiques et cognitives, mémoire d'habilitation à diriger les recherches)

PIGEOT N., VALENTIN B. 2003 - Les chronologies de la préhistoire dans le Bassin parisien au Tardiglaciaire : acquis récents, questions et bilan, in F. Widemann, Y. Taborin (dir.) Chronologies géophysiques et archéologiques du Paléolithique supérieur, Colloque du Centre européen de Ravello, mai 1994, p. 327-344.

VALENTIN B., PIGEOT N. 2000 - Éléments pour une chronologie des occupations magdaléniennes dans le Bassin parisien, in B. Valentin, P. Bodu, M. Christensen (éd.), L'Europe centrale et septentrionale au Tardiglaciaire, Actes de la Table-ronde internationale de Nemours (14-16 mai 1997), Edition Association pour la Promotion des Recherches archéologiques en Ile-de-France, Nemours, 7, p. $129-138$

PIGEOT N. (dir.) 2004 - Les derniers Magdaléniens d'Étiolles. Perspectives culturelles et paléohistoriques, XXXVIIe Supplément à Gallia Préhistoire, Éditions du CNRS, Paris, $345 \mathrm{p}$.

OLIVE M., PIGEOT N. 2006 - Réflexions sur le temps d'un séjour à Étiolles (Essonne) in M. Olive, B. Valentin (dir.), Variabilité des habitats tardiglaciaires dans le Bassin parisien et ses alentours - quelles significations?, Bulletin de la Société préhistorique française, 103, 4, p. 673-682.

PIGEOT N. 2011 - Chaînes opératoires : contexte théorique et potentiel cognitif, in R. Treuil (dir.) L'archéologie cognitive. Techniques, modes de communication, mentalités, Éditions Maison des Sciences de l'Homme, Paris, p. 149-171.

[1] Nous renvoyons à l'article d'hommage publié par Marianne Christensen, Carole Fritz, Dominique Legoupil, Monique Olive, Gilles Tosello et Boris Valentin dans le Bulletin de la société préhistorique française (Tome 117, numéro 1, janvier-mars 2020, p. 159-164) pour une bibliographie exhaustive. 